\title{
Calibration of Measuring Channels for the Analysis of the High-Power Microwave Impulse Parameters
}

\author{
Valery Galchenko \\ Applied Mathematics department \\ National Research Tomsk Polytechnic University \\ Tomsk, Russia \\ valery@tpu.ru
}

\author{
Tatyana Gladkova, Danila Bogdanov \\ Applied Mathematics department \\ National Research Tomsk Polytechnic University \\ Tomsk, Russia \\ gladkova@tpu.ru
}

\begin{abstract}
The calibration of measuring channels of the highpower microwave impulses, passing through various elements of the experimental setup, is an important task for quantifying the microwave impulse parameters during experimental research. The currently available calibration software packages have a significant disadvantage of having to enter various additional parameters within the software. This article presents the software package implemented in $Q T 4.5 \mathrm{C}++$ which significantly simplifies the input process of calibration data by utilizing the dialog mode for specifying the medium passage parameters of microwave impulses.
\end{abstract}

Keywords - calibration, measuring channels, microwave impulses, software, QT 4.5 C++ application framework, microwave impulse parameters.

\section{INTRODUCTION}

This paper describes the calibration of the frequencyindependent and frequency-dependent sensors used to study the parameters of high-power microwave impulses. The frequencydependent sensor includes beyond-cutoff attenuator and is used to evaluate the spectrum of the microwave impulses. The sensors are typically calibrated using special software packages designed in MathCad. These available calibration software packages have a common disadvantage of having to enter additional input parameters directly within the software, when the physical properties of the medium, which microwave impulses are traveling through, are changed.

This paper presents the sensors calibration software implemented in QT 4.5 C ++ [2]. Unlike other software packages implemented in MathCad, it allows all additional input parameters to be entered from a table generated from the input parameters file [3].

\section{MicrowaVe IMPULSE SENSORS CALIBRATION}

Fig. 1 below shows the schematics of the receiving sensors calibration stand. The microwave impulses from the calibration microwave generator operating in pulsed mode are transmitted via a coaxial-waveguide transition, tee, attenuators and other elements of the experimental setup towards the two microwave sensors - frequency independent sensor and frequency dependent sensor containing beyond-cutoff attenuator and a divider. At the divider the average power is measured with a standard meter. The calibration curves for sensors are then generated taking into account the deviation of the calibration impulse shape from the rectangular form.

During the calibration process impulses of 8 different power levels are generated utilizing microwave generator's attenuator ranging from 12 to $33 \mathrm{dBW}$. These power levels are approximately $15.625,31.25,62.5,125,250,500,1000,2000 \mathrm{~W}$ at frequencies ranging from $2700 \mathrm{MHz}$ to $3700 \mathrm{MHz}$ with 100 $\mathrm{MHz}$ increments (11 frequencies). The output voltage of the microwave impulses at these frequencies are recorded at both frequency-independent and frequency-dependent sensors using four-channel oscilloscope into two files, collecting 1001 samples at a time interval of 2 nanoseconds. Thus, during the calibration process a total of 88 files are recorded for each sensor which are then used to generate calibration curves. The use of 1001 samples greatly improves estimation accuracy of the calibration coefficients.

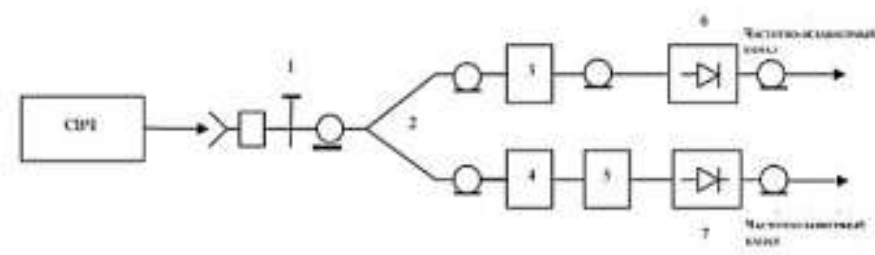

Fig. 1. Sensors Calibration Stand. 1- Coaxial-Waveguide Transition, 2 Splitter, 3,4 - Fixed Attenuator, 5 - Beyond-Cutoff Attenuator, 6,7 - Sensors.

\section{MATHEMATICAL DESCRIPTION OF CALIBRATION PROCESS}

Studies have shown [1], the calibration curves of linear (frequency-independent) and nonlinear (frequency-dependent) sensors can be accurately described by a 6-degree polynomial function where the coefficients on the 1,2,5,6 degree terms are substantially important whereas the coefficients on the third- and fourth-degree terms contribute no more than 1-2\%. Thus, the calibration polynomial for a certain frequency and a given impulse power level can be represented as follows: 


$$
F(x)=a_{1} \cdot x+a_{2} \cdot x^{2}+a_{5} \cdot x^{5}+a_{6} \cdot x^{6}
$$

$\alpha_{1}, \alpha_{2}, \alpha_{5}, \alpha_{6}-$ unknown coefficients, $x$ - sensor reading value at the frequency for which the calibration is performed.

Based on the experimental data the coefficients for calibration polynomial can be estimated utilizing the least squares method. The least squares function F1 for the frequency-independent sensor for each frequency is formulated as follows:

$$
F_{1}=\sum_{j=1}^{8} \sum_{i=1}^{1001}\left(U_{i l d}-\left(a_{1} x_{i}+a_{2} x_{i}^{2}+a_{5} x_{i}^{5}+a_{6} x_{i}^{6}\right)\right)^{2} \rightarrow \min
$$

$U_{i l d}$ - discrete voltage values on the microwave generator for a given frequency and power levels $j=1 \ldots 8$.

$\alpha_{1}, \alpha_{2}, \alpha_{5}, \alpha_{6}-$ sensor calibration coefficients for a given frequency and power level,

$i$-sample number for a given frequency and power level,

$x_{i}$ - value of the $i$ sample for a given frequency and power level,

The least square function F2 for the frequency-dependent sensor can be formulated in a similar way.

The function reaches its minimum value when partial derivatives of a function with respect to the unknown coefficients equal to zero:

$$
\frac{\partial F_{1}}{\partial a_{1 i}}=0 \quad \frac{\partial F_{1}}{\partial a_{2 i}}=0 \quad \frac{\partial F_{1}}{\partial a_{5 i}}=0 \quad \frac{\partial F_{1}}{\partial a_{6 i}}=0
$$

Consequently, the system of 4 linear equations to compute 4 calibration coefficients for each of the 11 frequencies for the linear sensor, is given by:

$$
\begin{aligned}
& \sum_{j=1}^{8} \sum_{i=1}^{1001} U_{i l d} x_{i}=\sum_{j=1}^{8} \sum_{i=1}^{1001}\left(a_{1} x_{i}+a_{2} x_{i}^{2}+a_{5} x_{i}^{5}+a_{6} x_{i}^{6}\right) \cdot x_{i} ; \\
& \sum_{j=1}^{8} \sum_{i=1}^{1001} U_{i l d} x_{i}^{2}=\sum_{j=1}^{8} \sum_{i=1}^{1001}\left(a_{1} x_{i}+a_{2} x_{i}^{2}+a_{5} x_{i}^{5}+a_{6} x_{i}^{6}\right) \cdot x_{i}^{2} ; \\
& \sum_{j=1}^{8} \sum_{i=1}^{1001} U_{i l d} x_{i}^{5}=\sum_{j=1}^{8} \sum_{i=1}^{1001}\left(a_{1} x_{i}+a_{2} x_{i}^{2}+a_{5} x_{i}^{5}+a_{6} x_{i}^{6}\right) \cdot x_{i}^{5} ; \\
& \sum_{j=1}^{8} \sum_{i=1}^{1001} U_{i l d} x_{i}^{6}=\sum_{j=1}^{8} \sum_{i=1}^{1001}\left(a_{1} x_{i}+a_{2} x_{i}^{2}+a_{5} x_{i}^{5}+a_{6} x_{i}^{6}\right) \cdot x_{i}^{6} .
\end{aligned}
$$

Solving this system of 4 linear equations results in $4 \times 11$ matrix of calibration coefficients - 4 coefficients for each of the 11 frequencies.

For nonlinear sensor the system of linear equations is formulated as follows:

$$
\begin{aligned}
& \sum_{j=1}^{8} \sum_{i=1}^{1001} U_{\text {inld }} y_{i}=\sum_{j=1}^{8} \sum_{i=1}^{1001}\left(b_{1} y_{i}+b_{2} y_{i}^{2}+b_{5} y_{i}^{5}+b_{6} y_{i}^{6}\right) \cdot y_{i} ; \\
& \sum_{j=1}^{8} \sum_{i=1}^{1001} U_{\text {inld }} y_{i}^{2}=\sum_{j=1}^{8} \sum_{i=1}^{1001}\left(b_{1} y_{i}+b_{2} y_{i}^{2}+b_{5} y_{i}^{5}+b_{6} y_{i}^{6}\right) \cdot y_{i}^{2} ; \\
& \sum_{j=1}^{8} \sum_{i=1}^{1001} U_{i n l d} y_{i}^{5}=\sum_{j=1}^{8} \sum_{i=1}^{1001}\left(b_{1} y_{i}+b_{2} y_{i}^{2}+b_{5} y_{i}^{5}+b_{6} y_{i}^{6}\right) \cdot y_{i}^{5} ; \\
& \sum_{j=1}^{8} \sum_{i=1}^{1001} U_{\text {inld }} x_{i}^{6}=\sum_{j=1}^{8} \sum_{i=1}^{1001}\left(b_{1} y_{i}+b_{2} y_{i}^{2}+b_{5} y_{i}^{5}+b_{6} y_{i}^{6}\right) \cdot y_{i}^{6} .
\end{aligned}
$$

Similarly, the calibration coefficients for the nonlinear sensor could be obtained by solving the system of linear equation given above (5).

\section{MiCROWAVE IMPULSE PARAMETERS EVALUATION}

The increase of the results handling efficiency has allowed to significantly expand capabilities of the experimental setup by introducing the mobile sensor. The mobile sensor allows to analyze the spatial distribution of the emission intensity by moving the sensor along the surface of an imaginary sphere with emitting generator window as a center, i.e., to measure the directional pattern generated by the microwave generator.

Fig. 2 below shows the schematics of the experimental setup for parameters evaluation of the microwave impulses traveled through the measuring channel. The signals at the experimental setup are registered by two four-channel digital oscilloscopes and are recorded into corresponding files. The obtained values are then processed utilizing the coefficients estimated depending on the measured parameters. In addition to the signals from the sensors the impulse current and the voltage at the microwave generator are also recorded. The Rogowski coil is used to register the triode currents.

The attenuation coefficients of the attenuators and other parameters of the experimental setup are stored in the initial data file, that can be viewed and updated if needed prior to processing. The changes are saved in the file and stored until the processing is completed. 


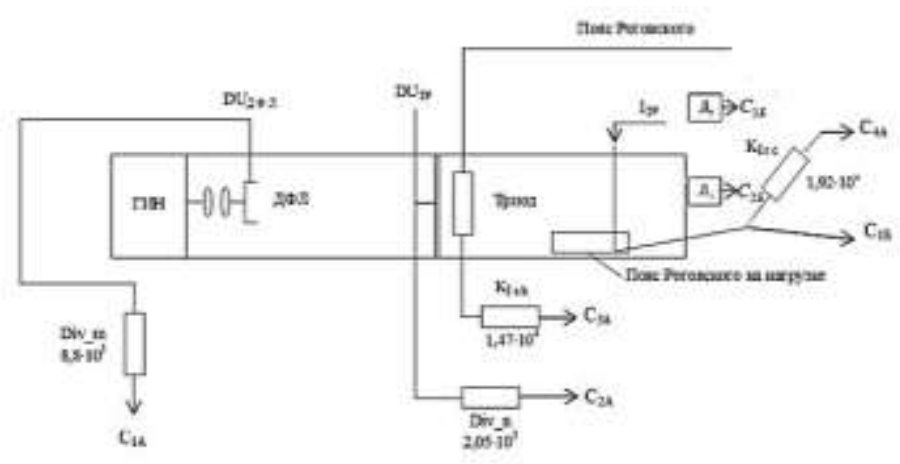

Fig. 2. Experimental Setup with Measuring Channels. ГИН - voltage impulse generator, ДФЛ - dual shaping line, Д1 - stationary sensor, Д2 -movable sensor, DUДФЛ - dual shaping line voltage, С1A (Um) - ГИН voltage (fed to channel 1 of oscilloscope A), DUTP - triode splitter voltage (fed to channel 2 of oscilloscope A (Un)), C2A - triode voltage (fed to channel 2 of oscilloscope A (Un), C3A - triode current (fed to channel 3 of oscilloscope A), C4A - Rogowski coil current (fed to channel 4 of oscilloscope A), С1Б - Rogowski coil current with triode under load (fed to channel 1 of oscilloscope A), ITP - triode current, Div_m - attenuator ГИН, Div_ $\mathrm{n}$ - diode voltage divider, KIsh - attenuator at the triode current circuit, KIrc - coefficient at the Rogowski coil current measuring circuit with triode under load, С2Б (U7) - stationary sensor voltage (fed to channel 2 of oscilloscope В), С ЗБ (U7) - mobile sensor voltage (fed to channel 3 of oscilloscope B).

\section{SOFTWARe PACKAGE Description}

The calibration software package is implemented in Qt 4.5 $\mathrm{C}++$ application framework. The designed software includes 5 modules, which subsequently provide the functionality of loading calibration files generated using digital oscilloscopes, computation of the 4 calibration coefficients for each of the 11 frequencies for both sensors, plotting calibration curves and saving calibration coefficients into two separate files. Obtained calibration coefficients are then used to calculate the parameters of the microwave impulse. The results of computation of the calibration coefficients for linear and non-linear sensors are presented in Fig. 3 and Fig. 4 below.

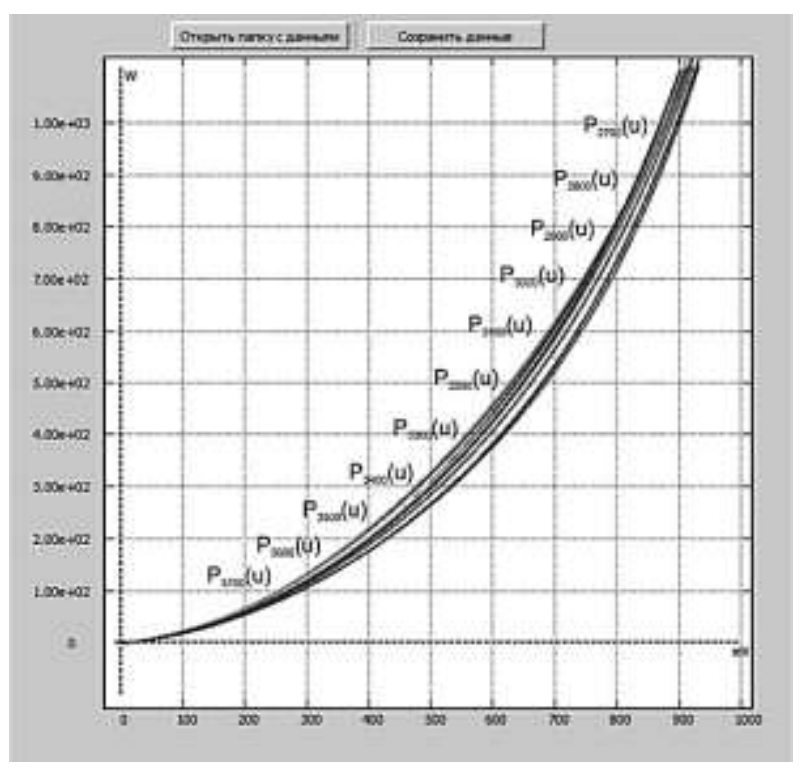

Fig. 3. Calibration Curves for Linear Sensor.

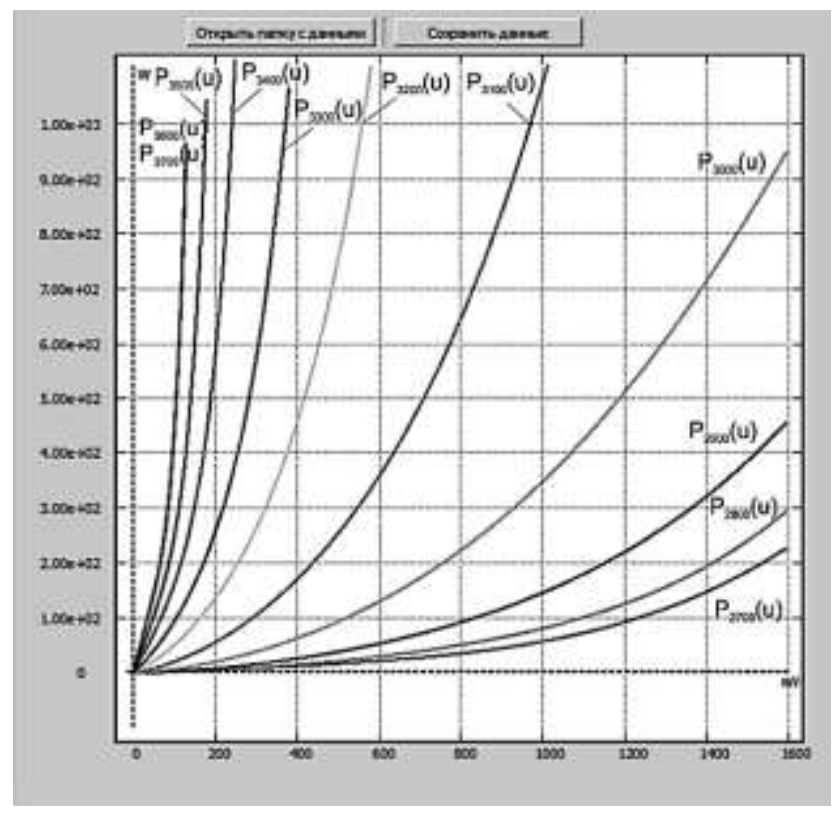

Fig. 4. Calibration Curves for Nonlinear Sensor.

During the experiment the signals registered by two fourchannel digital oscilloscopes are recorded into corresponding files and saved in two differed folders. The filename of each file contains the experiment number which simplifies files identification for further processing. Initial parameters required for experimental data processing are stored in the initial data file which is read at the start of the software. These values are then populated in the table. While processing the experimental results, the experimenter can change the input parameters when needed if 
the experimental conditions are changed. Upon reading of the experimental values the graphical representation is generated and values from the sensors are added. At the time when the spatial distribution of the emission intensity is studied an additional file is created containing the coordinates of the mobile sensor.

After all data from the sensors is processed taking into account the calibration coefficients the plots are generated. Each plot has the capability for detailed investigation of any specific area. For that purpose, a temporary time cutoff can be applied by selecting the initial and final cut-off points.

The quantitative evaluation of the parameters is performed utilizing the moving markers.

In order to assess the current value of the microwave impulse amplitude and other parameters of the experimental setup the first moving marker can be moved along the curve and a corresponding quantitative value will be displayed in a separate window.

In addition, the plot allows to read the time delay between individual parameters by utilizing the second moving marker. The position of the second moving marker determines the time delay between the first and the second moving marker, and the value of the time delay is displayed in a separated window.

Fig. 5 shows the plot of several parameters of the experimental setup and a certain shape of the microwave impulse obtained by utilizing the stationary and mobile sensors.

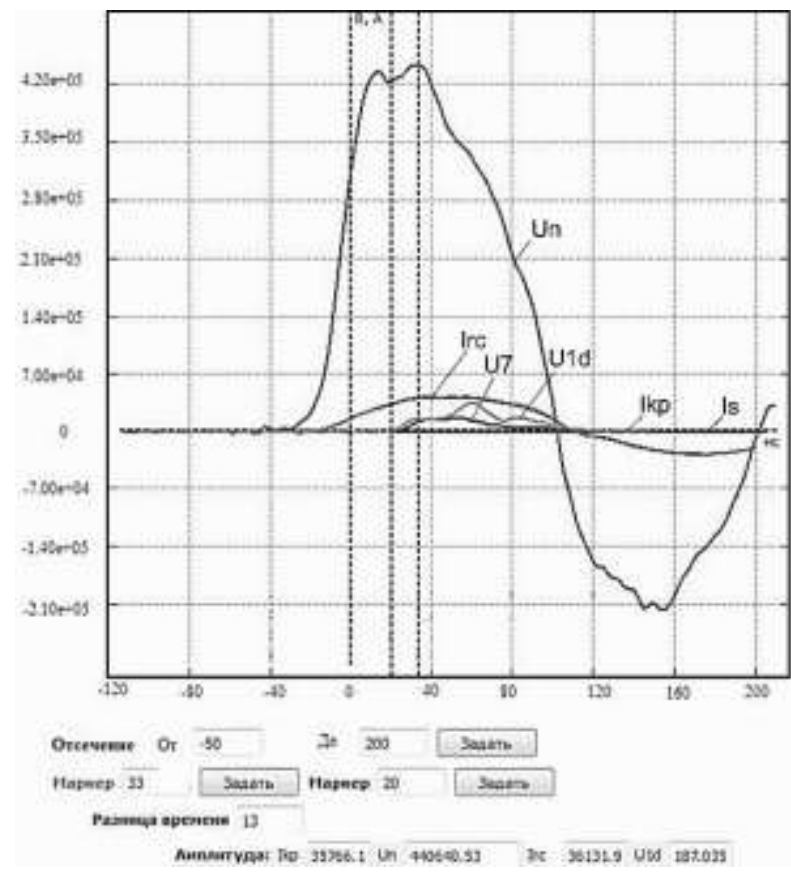

Fig. 5. Sample of the Microwave Impulse and Other Parameters of the Experimental Setup. Ikp - Rogowski coil current, Is (C3A) - triode current, Un (C2A) - triode voltage, U7 (C2Б) - stationary sensor voltage, Irc (C4A) Rogowski coil current under load, U1d (С4Б) - mobile sensor voltage.

\section{CONCLUSION}

The actual implementation of the calibration procedure described in this paper, did not reveal any issues while using the designed software neither during the calibration stage nor subsequent evaluation of the parameters of high-power microwave impulses.

\section{REFERENCES}

[1] Babichev D.A., Shijan V.P.,Melnikov G.V. Measuring device of frequency composition of high-power microwave impulses of nanosecond duration. Equipment and technique, №3, (2003) p. 93-96.

[2] Qt4.5 C++. Professional programming in $\mathrm{C}++.-\mathrm{SPb}$. : BHB - Peterburg, (2010) .- p896.

[3] Kosicin V.S., Galchenko V.G., Gladkova T.A. Software package for parameters evaluation of high-power microwave impulses. // Tomsk Polytechnic University News. - 2013, v. 322. - № 5, p. 205-208. 